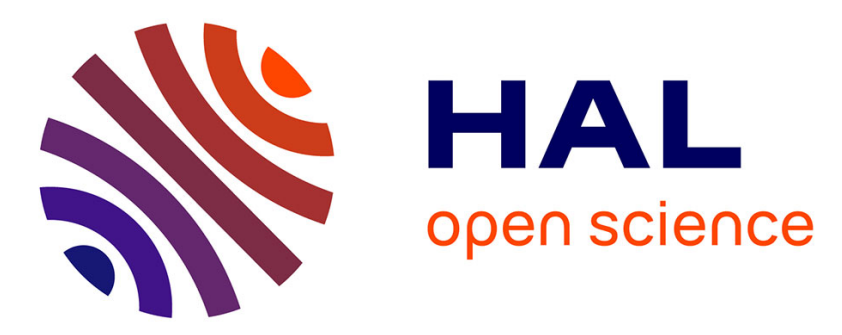

\title{
L'orthopédie pédiatrique en milieu précaire. Possibilités et limites de la chirurgie humanitaire
}

Michel Onimus, Bertrand Tekpa

\section{To cite this version:}

Michel Onimus, Bertrand Tekpa. L'orthopédie pédiatrique en milieu précaire. Possibilités et limites de la chirurgie humanitaire. Revue de Chirurgie Orthopédique et Traumatologique, 2019, 105, pp.243 - 247. 10.1016/j.rcot.2019.01.018 . hal-03486470

\section{HAL Id: hal-03486470 https://hal.science/hal-03486470}

Submitted on 20 Dec 2021

HAL is a multi-disciplinary open access archive for the deposit and dissemination of scientific research documents, whether they are published or not. The documents may come from teaching and research institutions in France or abroad, or from public or private research centers.
L'archive ouverte pluridisciplinaire HAL, est destinée au dépôt et à la diffusion de documents scientifiques de niveau recherche, publiés ou non, émanant des établissements d'enseignement et de recherche français ou étrangers, des laboratoires publics ou privés.

\section{다(1) $\$$}

Distributed under a Creative Commons Attribution - NonCommercial| 4.0 International 


\title{
Mémoire Original - Orthopédie humanitaire
}

\author{
L'ORTHOPEDIE PEDIATRIQUE EN MILIEU PRECAIRE. \\ POSSIBILITES ET LIMITES DE LA CHIRURGIE HUMANITAIRE ${ }^{1}$ \\ Pediatric orthopedic in precarious environment. Possibilities and limits of \\ humanitarian surgery
}

Michel ONIMUS ${ }^{a}$, Bertrand TEKPA ${ }^{\text {b }}$

a. Faculté de Médecine de Besançon, Université de Franche-Comté, 25000 Besançon, France

b. Service d'Orthopédie-Traumatologie, $\mathrm{CHU}$ Communautaire, Avenue des Martyrs, BP 2535, Bangui, République centraficaine

\section{Auteur correspondant : Michel Onimus}

Faculté de Médecine de Besançon - Université de Franche-Comté FRANCE monimus@wanadoo.fr

\section{RESUME}

\section{INTRODUCTION}

Cette étude rétrospective rapporte l'expérience de 35 ans de chirurgie humanitaire en orthopédie pédiatrique réalisée dans des conditions précaires.

\section{MATERIEL ET METHODES}

L'étude porte sur 78 missions chirurgicales réalisées en République Centrafricaine depuis 1983. Les missions se déroulaient dans la capitale et dans une quinzaine de localités où existaient de petits centres de rééducation créés lorsque la poliomyélite antérieure aiguë faisait des ravages. La chirurgie se déroulait dans le centre de santé ou l'hôpital local avec la participation du personnel local, parfois sans électricité, souvent sans radiographie, obligeant à des diagnostics purement cliniques.

RESULTATS

7000 enfants ont été vus en consultation et 2100 ont été opérés. Les séquelles de poliomyélite qui représentaient $66 \%$ des consultants entre les années 1983 et 2000 ,

\footnotetext{
${ }^{1}$ Cet article est consultable sur www.sciencedirect.com et sur www.emc-consulte.com/produit/rcot
} 
sont devenues peu fréquentes, remplacées par une pathologie variée dans laquelle prédominent les malformations congénitales et les séquelles d'injection intramusculaires, de même que les séquelles de brûlures et les séquelles neurologiques (IMOC, séquelles de neuropaludisme, séquelles d'intoxication par du manioc non détoxifié).

\section{DISCUSSION}

Le peu de coopération des familles explique que seulement $25 \%$ des enfants opérés aient pu être revus, empêchant toute analyse statistique des résultats. Néanmoins de nombreuses informations concernant l'évolution postopératoire ont pu être obtenues et permettent une appréciation globale du bien-fondé de ce type de chirurgie humanitaire.

Mots clés: orthopédie pédiatrique, poliomyélite antérieure aigue; chirurgie humanitaire ; séquelles d'injection intramusculaire ; chirurgie orthopédique en milieu précaire.

\section{INTRODUCTION}

La chirurgie humanitaire peut s'effectuer dans des conditions très variables, tenant surtout au contexte dans lequel elle est exercée. A côté de la chirurgie d'aide en urgence, la chirurgie d'aide au développement peut prendre plusieurs formes selon les lieux où elle est pratiquée et les moyens dont elle dispose. A partir d'une expérience de 35 ans de chirurgie orthopédique pédiatrique en milieu précaire, les auteurs analysent la pathologie observée et les possibilités de prise en charge, ainsi que les résultats obtenus.

\section{MATERIEL D'ETUDE}

II s'agit d'une étude rétrospective portant sur 78 missions chirurgicales réalisées en République Centrafricaine depuis 1983. L'objectif initial était la prise en charge des séquelles de poliomyélite antérieure aiguë. Ces missions se déroulaient de façon décentralisée, pour moitié dans la capitale où le plateau technique est correct, et pour moitié en province dans une quinzaine de localités pourvues d'un centre de rééducation et appareillage (créés dans les années 1970-80 lorsque la poliomyélite était endémique). Les personnels de ces centres (religieuses missionnaires, rééducateurs) étaient nos indispensables partenaires locaux; ils effectuaient le 
dépistage des enfants, les rassemblaient pour la mission chirurgicale, et assuraient la rééducation postopératoire ainsi que l'appareillage.

La chirurgie se déroulait dans les structures de santé locales (hôpital, centre de santé...) en apportant le consommable nécessaire et en étroite collaboration avec le personnel médical pour lequel un important effort de formation a été fait. Le principe était de faire avec les moyens disponibles localement, avec des protocoles simples et reproductibles, donc sans apporter de moyens et de techniques sophistiqués, et en prévoyant des soins postopératoires simples, limités le plus possible à l'ablation du plâtre et aux pansements. En l'absence de possibilités d'ostéosynthèse, le maintien des corrections opératoires était confié à l'immobilisation plâtrée et la confection des plâtres postopératoires était un temps important de l'acte chirurgical. Chaque fois que cela était possible une hémostase par bande d'Esmarch était réalisée. Les anesthésies générales étaient faites avec des protocoles locaux utilisant Diazépam, Kétamine, Atropine, sans intubation (sauf pour la chirurgie des fentes labiales) ; les indications de l'anesthésie caudale étaient élargies jusqu'à l'âge de 6 ou 7 ans et la rachianesthésie à partir de l'âge de 12 à 13 ans. En province en particulier les conditions locales étaient souvent précaires: parfois pas d'électricité, radiographies rarement disponibles obligeant à des diagnostics essentiellement cliniques.

Durant cette expérience de presque 35 ans, 7000 enfants ont été vus en consultation et 2100 ont été opérés. Les séquelles de poliomyélite qui représentaient $67 \%$ des consultants entre les années 1983 et 2000 et $70 \%$ des interventions, sont devenues très peu fréquentes depuis la diffusion des campagnes de vaccination (7\% entre 2000 et $2010,2 \%$ entre 2010 et 2017) ; les pathologies rencontrées depuis 2010 étaient plus variées (Encadré 1).

Les interventions les plus fréquentes depuis cette date portaient sur les malformations congénitales (33\%) et sur les séquelles d'injections intramusculaires (15\%) (Encadré 1).

\section{RESULTATS}

Quatre décès sont survenus à la suite d'anesthésies effectuées dans des conditions précaires. Des complications septiques ont été notées essentiellement dans 2 circonstances : après double arthrodèse sous-talienne et médiotarsienne ( 9 cas sur 154 soit $6 \%$ ), et après désinsertion large du quadriceps (23 cas sur 149 , soit $15 \%$ ). 
Du fait des conditions de réalisation des missions et du fait du peu de coopération des familles, seuls $20 \%$ à $25 \%$ des enfants opérés ont pu être revus ; cependant des informations nous ont été transmises dans de nombreux cas, permettant d'avoir une appréciation globale du travail effectué.

3.1 Les séquelles de poliomyélite antérieure aiguë, qui représentaient l'essentiel de l'activité jusqu'aux années 2000, sont devenues peu fréquentes. Les protocoles chirurgicaux utilisés sont simples : ténotomie des fléchisseurs de hanche (sartorius, droit fémoral, tenseur du fascia lata poursuivie sur le fascia lata selon l'importance de l'abductum de la hanche), ténotomie des fléchisseurs du genou incluant toujours le fascia lata, et allongement du tendon d'Achille ou double arthrodèse sous talienne et médiotarsienne. La rééducation postopératoire obéit à un protocole rigoureux : le flexum de hanche est très rapidement corrigé par mise en posture en décubitus ventral dès la fin de l'intervention; Le flexum de genou est corrigé par des attelles articulées verrouillables qui permettent la correction progressive du flexum et la mobilisation quotidienne du genou. Avec ces protocoles on obtient une correction complète des rétractions quel que soit leur gravité tout en conservant les amplitudes articulaires. Nous avions obtenu $70 \%$ de résultats satisfaisants, évalués sur la capacité pour l'enfant d'aller à l'école [1].

3.2 Les malformations congénitales sont très fréquentes, et particulièrement le pied bot varus équin congénital (Encadré 2). La libération postéro-médiale est rendue difficile par la raideur et l'importance des déformations; la réduction est souvent incomplète malgré une large libération et notamment une capsulotomie de tout l'interligne sous-talien médial. La mise en route du protocole de Ponseti il y a une dizaine d'années a nettement facilité le traitement. Cependant le traitement précoce selon Ponseti, réalisé dès les premiers mois, nous a déçus, surtout en raison du manque de coopération des familles qui supportent mal une contention prolongée, et il nous a semblé préférable de retarder le début du traitement jusqu'aux mois précédant l'acquisition de la marche, pour que le traitement soit achevé au moment de la verticalisation, celle-ci apportant un facteur de stabilité au résultat. Une libération postéro-médiale pouvait être nécessaire, mais elle était toujours considérablement facilitée par l'assouplissement obtenu par les plâtres successifs. Une talectomie a été réalisée dans 7 cas de déformations rigides chez des enfants âgés de 5 à 9 ans. Le résultat clinique était correct avec un appui au sol plantigrade, mais la marche restait lente et sans déroulement du pas. 
3.3 Les séquelles d'injection intramusculaire sont une pathologie iatrogène endémique qui représente actuellement $16 \%$ des consultations. L'injection de sels de quinine est le traitement le plus utilisé pour le traitement de l'accès palustre, car le moins coûteux et le plus facilement disponible. Cependant une injection mal exécutée et trop médiale dans la fesse peut entrainer une paralysie partielle du nerf sciatique, avec atteinte du contingent du nerf fibulaire commun responsable d'une déformation du pied en varus équin. Le muscle tibial postérieur reste fonctionnel et transposable sur le dos du pied. Une déformation en varus équin irréductible nécessite une double arthrodèse sous-talienne et médiotarsienne à laquelle on peut associer une transposition du tibial postérieur [2]. Les résultats fonctionnels de la transposition du tibial postérieur ont pu être évalués sur 30 cas revus : ils étaient bons dans 50\% des cas, avec un transplant efficace permettant d'amener le pied à l'angle droit. Si l'injection intra-fessière est trop profonde, elle peut se faire dans l'articulation de la hanche et provoquer une nécrose de la tête fémorale, dont nous avons observé plusieurs cas documentés par une radiographie (fig 1). Chez l'enfant jeune, l'injection est faite dans le quadriceps sur la face antérieure de la cuisse, et elle peut entrainer une fibrose rétractile du muscle. Parfois la rétraction est limitée aux fibres du muscle droit antérieur fémoral avec une raideur du genou apparente en extension de la hanche et avec une simple gêne lors du pas postérieur. Plus souvent l'injection est responsable d'un enraidissement du genou en extension complète, parfois même d'un recurvatum irréductible (fig 2). Le handicap est important surtout lorsque l'injection est bilatérale. Le traitement impose une désinsertion large du quadriceps selon Judet [3], nécessitant de disposer d'un bistouri électrique. La rétraction s'étend souvent jusqu'aux fibres les plus médiales du muscle vaste médial dont la libération doit être prudente à cause du risque vasculaire. On obtient habituellement une flexion complète du genou, mais le résultat à plus long terme dépend de la qualité de la rééducation postopératoire et dans plus de la moitié des cas revus nous avons observé une perte secondaire significative avec une amplitude résiduelle de $30^{\circ}$ à $60^{\circ}$, permettant néanmoins un déroulement normal du pas. Malgré une hémostase soigneuse, un hématome postopératoire secondairement infecté est survenu dans $15 \%$ des cas et a compromis le résultat fonctionnel. La quadriceps-plastie proximale [4] ou distale [5] a été proposée comme alternative à la désinsertion du muscle; cependant cela oblige à une immobilisation de quelques 
semaines en extension, et surtout la rétraction des fibres du vaste médial peut rendre peu efficace ce procédé qui s'applique surtout aux rétractions du droit fémoral 3.4 Les brûlures sont très fréquentes, car le feu de cuisson du repas familial est fait par terre et les enfants, surtout épileptiques, tombent facilement dedans. Les séquelles sont particulièrement fréquentes au niveau de la main avec des rétractions cutanées qui limitent le jeu articulaire. La perte de substance obtenue après section de la bride cutanée est couverte par un greffon de peau totale prélevé sur l'abdomen. La suture de la zone du prélèvement évite des pansements ultérieurs répétés, douloureux et coûteux.

Les déviations axiales des membres inférieurs en genu valgum ou genu varum sont fréquentes et d'étiologie imprécise, peut-être liées à un rachitisme méconnu, aggravé par des facteurs nutritionnels (en Centrafrique l'aliment de base est le manioc, connu pour être sans aucune valeur nutritionnelle). Dans quelques cas de genu varum une radiographie a permis le diagnostic de maladie de Blount, incitant à réaliser une hypercorrection en raison du risque de récidive. Le genu valgum était corrigé par ostéotomie fémorale sus-condylienne et le genu varum par ostéotomie tibiale soustubérositaire associée à une ostéotomie de la fibula (fig 3a et b). En l'absence d'ostéosynthèse la correction était maintenue par un plâtre cruro-pédieux. Le petit nombre de cas revus et documentés (une vingtaine sur 80 ostéotomies) ne permet pas d'avoir de données chiffrées concernant les résultats postopératoires.

3.5 Les séquelles motrices d'atteinte cérébrale étaient fréquentes $115 \%$ des consultants) ; il s'agissait soit de paralysie cérebrale par atteinte périnatale, soit de séquelles de méningite ou de neuropaludisme avec souvent un déficit intellectuel ; dans quelques cas ces séquelles ont été rattachées à une intoxication par du manioc mal rouï (maladie du Konzo [6-7]), donnant un tableau de paraparésie spasmodique d'apparition brutale; le manioc consommé en Centrafrique contient en effet du cyanure qui doit être éliminé par rouïssage dans l'eau pendant quelques jours, puis par séchage. Nous avons observé ces séquelles dans quelques localités dans des périodes de disette où le manioc était consommé trop rapidement. Dans tous ces cas d'atteinte centrale, les possibilités thérapeutiques se limitaient à des ténotomies qui amélioraient la marche, celle-ci restant néanmoins le plus souvent tributaire de cannes ou béquilles.

3.6 Les séquelles d'infection ostéo-articulaire revêtaient plusieurs aspects. Dans 20 cas un séquestre était visible et le traitement était facile. Dans 34 cas une 
radiographie révélait la présence d'un séquestre dont l'ablation amenait la guérison. Le traitement des inégalités de longueur dues à une perte de substance diaphysaire ou à une stérilisation du cartilage conjugal était impossible en l'absence d'instrumentation spécifique. Des ankyloses articulaires en position vicieuse ont été corrigées au niveau des articulations périphériques.

3.7 La prise en charge des fentes labiales est récente, le premier cas ayant été opéré en 2006. Les fissures labiales se sont révélées être une pathologie relativement fréquente, mais ignorée, les enfants restant confinés dans la case et peu intégrés dans le groupe social. La technique utilisée était celle de Delaire [8], simple et facile à mettre en pratique. La chirurgie nécessitait une intubation, limitant sa réalisation à des centres équipés en oxygène. Les suites opératoires ont été simples dans tous les cas, malgré un environnement peu respectueux des consignes (les enfants commençaient parfois à manger dès le lendemain de l'intervention...).

Les lésions traumatiques ont été vues secondairement au stade de séquelles; elles ont rarement donné lieu à une indication chirurgicale, car les déformations étaient tolérables avec un bon pronostic de remodelage, ou par manque de moyens (traumatismes du rachis notamment).

\section{DISCUSSION}

L'orthopédie infantile ne travaille pas dans l'urgence ; la chirurgie est programmée et elle ne peut se faire qu'avec des partenaires locaux qui préparent les missions, recensent les enfants, les rassemblent au moment de la mission et assurent les soins postopératoires. II n'existe pas de politique de dépistage précoce et les déformations observées sont habituellement accentuées et peu réductibles, rendant la chirurgie difficile. Par ailleurs les familles suivent peu les prescriptions ; les rendezvous sont souvent oubliés, notamment les rendez-vous de suivi.

L'avantage principal de travailler de façon décentralisée est de simplifier l'accès aux soins pour les familles, en leur évitant des déplacements compliqués (en Afrique, la mère reste auprès de l'enfant durant toute son hospitalisation, et elle doit donc abandonner la culture des champs et l'entretien de la famille durant parfois plusieurs semaines). L'inconvénient de ce type de chirurgie est l'impossibilité de mettre en route des protocoles longs comme la chirurgie d'égalisation des membres. Malgré le caractère habituellement sommaire des locaux opératoires et des conditions d'asepsie très rudimentaires, les complications septiques ont été peu fréquentes ; ceci s'explique d'une part par des interventions courtes et le plus souvent peu 
agressives et d'autre part par la pratique d'un « no touch technique» strict. Un gros effort d'enseignement a été réalisé, mais avec le recul, force est de reconnaitre l'échec quasi-total de cette formation, peut-être par manque d'intérêt des professionnels de santé.

\section{CONCLUSION}

L'orthopédie pédiatrique est une spécialité encore peu pratiquée dans les pays en développement, peut-être en partie en raison du système du recouvrement des coûts qui impose aux familles de financer en totalité l'ensemble des frais de prise en charge de l'enfant; or le handicap d'un enfant n'est pas considéré comme une priorité car ne présentant pas de risque vital, mais plutôt comme une fatalité. Néanmoins les besoins sont importants; les lésions de l'appareil locomoteur de l'enfant sont fréquentes, souvent très handicapantes, et beaucoup de ces lésions sont accessibles à un traitement efficace que l'on peut réaliser avec des techniques simples même dans des conditions sommaires.

\section{Conflits d'Intérêts : aucun}

Financement : aucun

Contribution des auteurs : Les auteurs ont contribué également à la rédaction de l'article.

\section{REFERENCES}

1. ONIMUS M, MANDABA JL. Les possibilités de verticalisation et de marche chez l'enfant porteur de séquelles de poliomyélite en milieu africain (Walking in Children after Poliomyelitis). Intern Orthop (SICOT) 1992 ; 16 : 196-201.

2. ONIMUS M, BRUNET L, GAUDEUILLE F, ISSA-MAPOUKA A. Le traitement des séquelles d'injections intramusculaires de sels de quinine en milieu africain. Med Trop $2007 ; 67:$ 267-73.

3. JUDET J, JUDET R, LAGRANGE J. Une technique de libération de l'appareil extenseur dans les raideurs du genou. Mem Acad Chir 1956 ; 82 : 944-7.

4. SENGUPTA S. Pathogenesis of infantile quadriceps fibrosis and its correction by proximal release. J Pediatr Orthop $1985 ; 5: 187-91$. 
5. SOUMAH MT, SYLLA AL, TOURE MR et al - Fibrose quadricipitale après injections intramusculaires dans la cuisse : à propos de 92 cas à l'Hôpital central universitaire Ignace Deen à Conakry. Med Trop 2003; 63 : 49-52.

6. Tylleskär T, Banea M, Bikangi N, Cooke RD, Poulter NH, Rosling H. Cassava cyanogens and konzo, an upper motoneuron disease found in Africa. Lancet. 1992; $339: 208-11$.

7. Tylleskär T, Légué FD, Peterson S, Kpizingui E, Stecker P. Konzo in the Central African Republic. Neurology. 1994; 44 : 959-61.

8. MARKUS AF, DELAIRE J. Functional primary closure of the cleft lip. British Journal of oral and maxillofacial surgery $1993 ; 31: 281-291$. 
Encadré 1. Les pathologies rencontrées entre 2010 et 2017 (sur 2289 consultants)

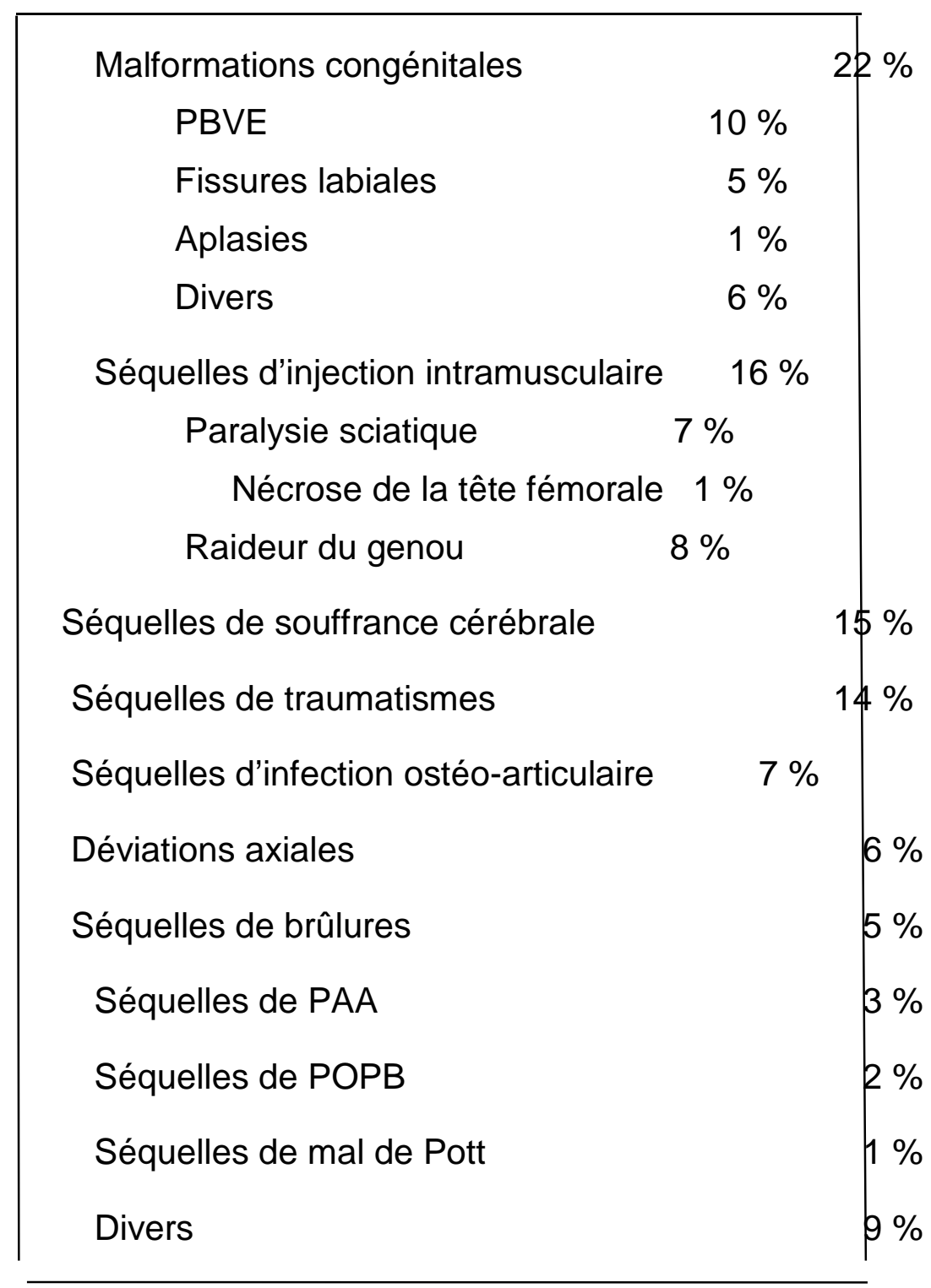

Malformations congénitales diverses : syndactylies, hypertrophies congénitales, spina bifida, mains botes radiales...

Déviations axiales : genu varum, genu valgum, incurvations osseuses

PBVE : pied bot varus équin

$P A A$ : poliomyélite antérieure aigue

POPB : paralysie obstétricale du plexus brachial 
Encadré 2. Les interventions pratiquées depuis 2011 (sur 730 opérations)

\begin{tabular}{|lcc|}
\hline Pied bot varus équin & & $25 \%$ \\
Libération postéro-interne & $11 \%$ & \\
Protocole de Ponseti & $6 \%$ & \\
Double arthrodèse & $7 \%$ & \\
Talectomie & $1 \%$ & \\
Fissures labiales & & $8 \%$ \\
Malformations congénitales diverses & $5 \%$ \\
Séquelles d'injection intramusculaire & $15 \%$ \\
$\quad$ Transposition du Tibial Post & $6 \%$ & \\
$\quad$ Désinsertion du quadriceps & $9 \%$ & \\
Ostéotomies pour déviations axiales & & $11 \%$ \\
Ténotomies pour spasticité & & $11 \%$ \\
Séquelles de brûlures & $10 \%$ \\
Ostéites chroniques et séquelles & $4 \%$ \\
Séquelles de traumatismes & $1 \%$ \\
Séquelles de PAA & $2 \%$ \\
Divers & $8 \%$ \\
\hline
\end{tabular}


Figure 1. Nécrose de la tête fémorale chez un enfant âgé de 13 ans, avec une notion d'injection intra-fessière réalisée 4 ans auparavant, ayant fait porter le diagnostic de nécrose post-injectionnelle.

Figure 2. Important genu recurvatum observé après injection intraquadricipitale de quinimax.

Figure 3. Déviation en coup de vent. a) aspect préopératoire ; b) aspect après ostéotomie fémorale du côté du valgus et ostéotomie tibiale du côté du varus. 


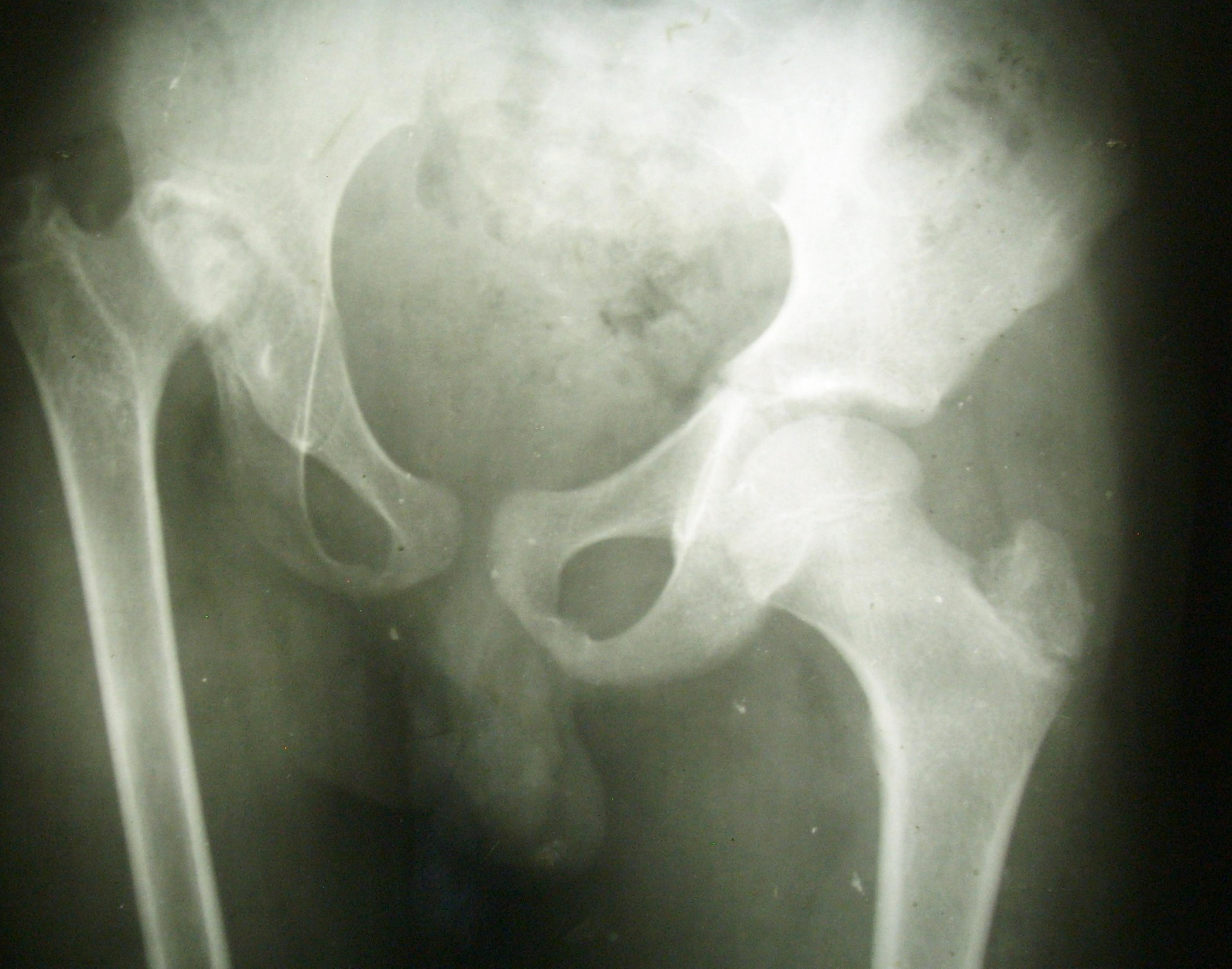




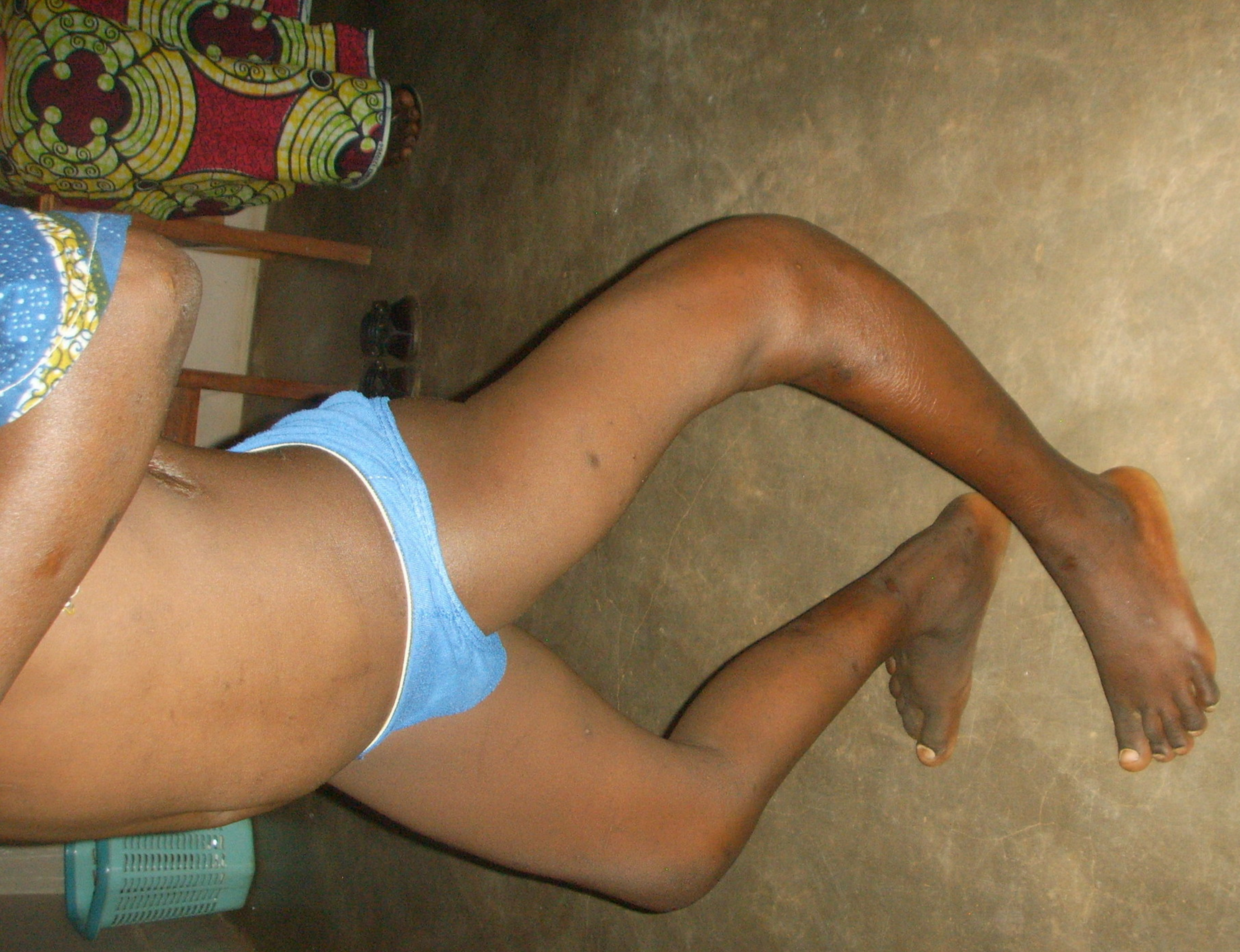




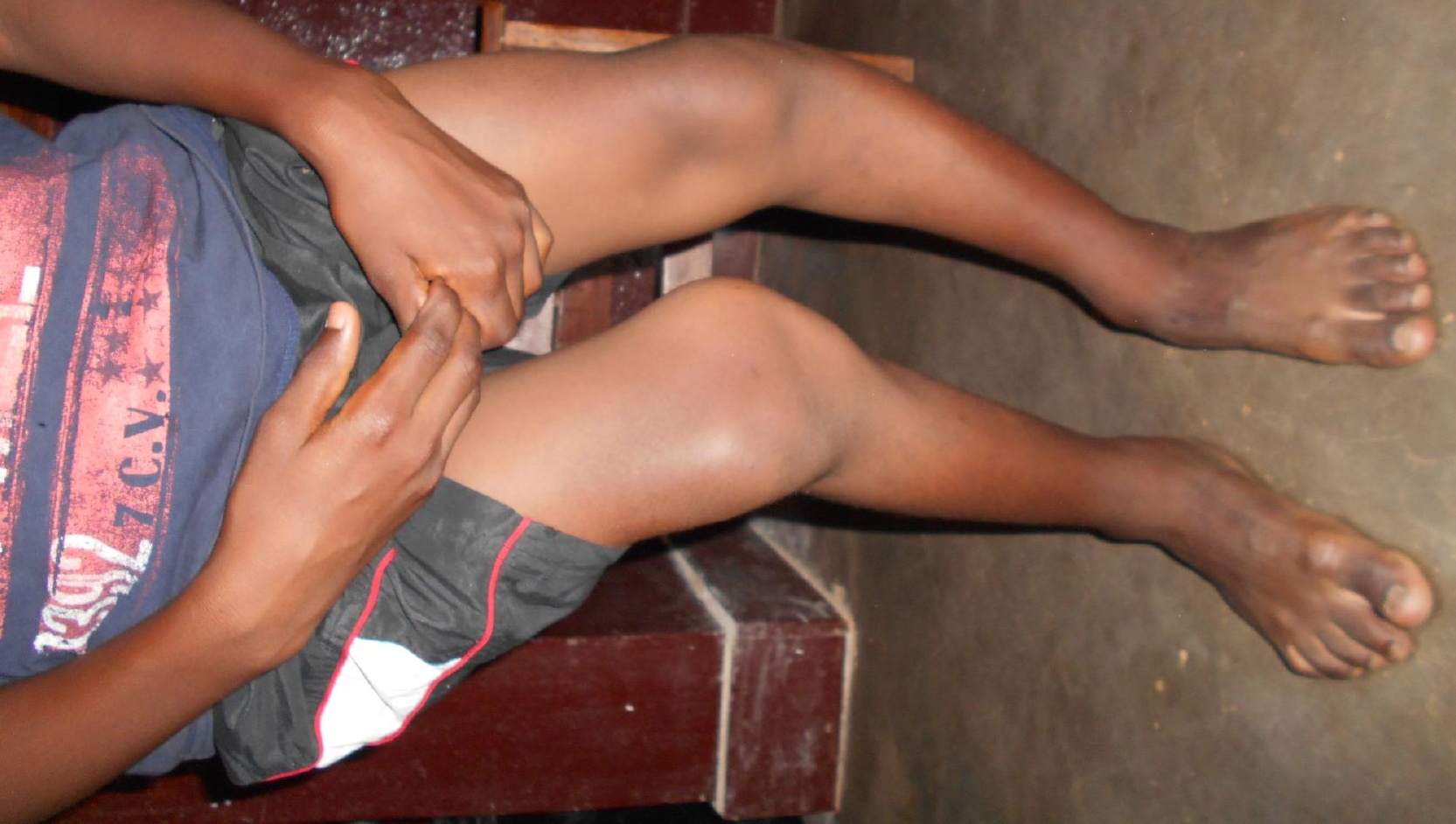




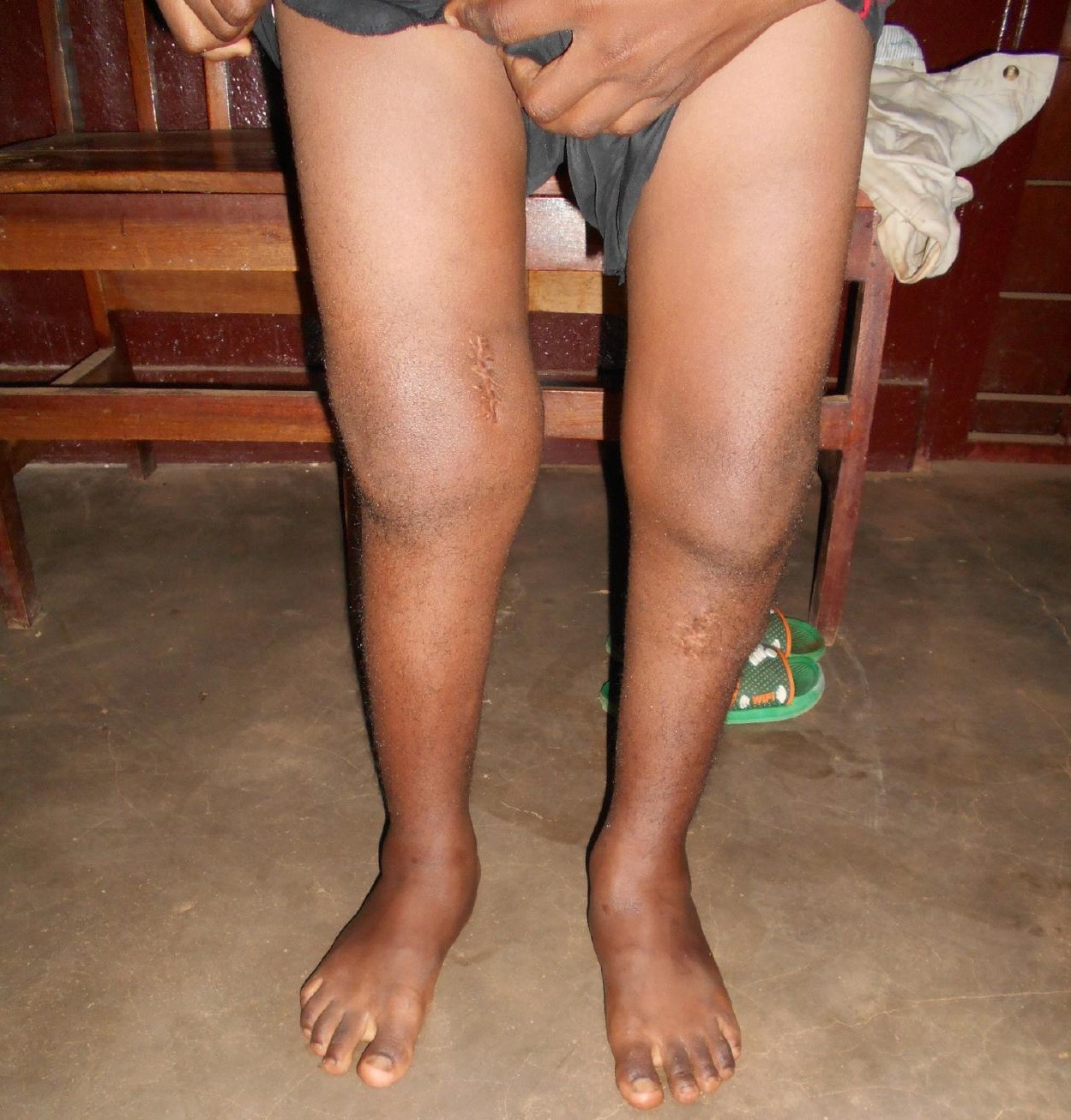

